\title{
Correspondence
}

\section{An unusual occurrence in transfusion of autologous whole blood and Pentaspan®. Beware!}

To the Editor:

Within a two-week period our Transfusion Medicine Service was notified of difficulties in the transfusion of autologous whole blood in the surgical suite for two separate patients. ${ }^{1}$ Both of these patients participated in pre-donation of autologous whole blood in our hospital-based autologous program. Both of these patients had received Pentaspan ${ }^{\circledR}$ immediately preceding the transfusion of pre-stored autologous whole blood via the standard Y-administration set for blood with 170 micron filter. The transfusion of the pre-stored autologous whole blood immediately following Pentaspan ${ }^{\circledR}$ resulted in the prompt formation of a thick gelatinous "clot" in the filter portion of the $i v$ administration tubing set in both of these cases.

Our Transfusion Medicine Service contacted Canadian Blood Services, Bristol-Myers-Squibb (marketer of Pentaspan ${ }^{\circledR}$ in Canada) and several Transfusion Medicine experts and conducted a literature review. An internal audit of autologous whole blood collection volumes as determined by unit mass was also conducted and found to be within acceptable limits as described by the Draft Standards for Blood Safety (Health Canada) as circulated at the time (2002). Other than the awareness of Pentaspan ${ }^{\circledR}$ being a rouleaux-causing agent,${ }^{2}$ there were no specific case reports of a similar phenomenon.

Subsequently, our Transfusion Medicine Service conducted an in vitro experiment where we passed autologous whole blood, red cell concentrate leukoreduced, and fresh frozen plasma leukoreduced through standard Y-administration sets with 170 micron filter following a flush with Pentaspan ${ }^{\circledR}$. No clot formation occurred when leukoreduced red cell concentrate and fresh frozen plasma were infused following Pentaspan ${ }^{\circledR}$. When stored autologous whole blood was infused following Pentaspan ${ }^{\circledR}$, there was immediate formation of gel-like clotted material within the filter portion of the administration set. Histological examination revealed a fibrin clot with white cells and platelets.

In conclusion, Pentaspan ${ }^{\circledR}$ appeared to interact with stored non-leukoreduced autologous whole blood to form a fibrin clot that could possibly be asso- ciated with plasma, platelets or white cells of stored autologous whole blood. This fibrin clot formation may prohibit the transfusion of the recipient's autologous blood and could delay therapy. Autologous whole blood should be treated as any other blood component and only combined or transfused with $0.9 \%$ sodium chloride for administration and caution should be exerted in transfusing stored autologous whole blood via an administration set that has been used to infuse Pentaspan®.

Lois Shepherd MDCM FRCPC

Doris Flynn RN BSCN ENC(C)

Ann-Marie Smith MLT BSc

Kingston General Hospital, Kingston, Canada

E-mail: shepherd@cliff.path.queensu.ca

\section{References}

1 Shepherd L, Flynn DJ, Smith AM. An unusual adverse effect associated with the transfusion of autologous whole blood and Pentaspan®. Beware! Transfusion 2003; 43(Suppl 9): 112A-3A (abstract).

2 Denesiuk L, Clarke G. Effect of pentastarch on routine blood bank tests. Transfusion 2003; 43(Suppl 9): 101A (abstract).

\section{Antiemetic efficacy of propofol at small doses for reducing nausea and vomit- ing following thyroidectomy}

To the Editor:

Postoperative nausea and vomiting (PONV) are common and unpleasant complications following thyroidectomy. ${ }^{1}$ Propofol, in small doses, possesses direct antiemetic properties. ${ }^{2}$ We previously reported that a single small dose $\left(0.5 \mathrm{mg} \cdot \mathrm{kg}^{-1}\right)$ of propofol administered intravenously at the end of surgery was more effective than traditional antiemetics, droperidol and metoclopramide, for preventing PONV in patients scheduled for thyroidectomy. ${ }^{3}$ In the present study, we determined the minimum effective dose of propofol for prophylaxis against PONV in this population.

Eighty patients ( 62 females), aged 20 to 68 yr scheduled for thyroidectomy under general anesthesia were enrolled. Anesthesia was induced with thiopentone $\mathbf{5}$ $\mathrm{mg} \cdot \mathrm{kg}^{-1} i v$ and fentanyl $2 \mu \mathrm{g} \cdot \mathrm{kg}^{-1} i v$, with tracheal intu- 